\title{
EARNING QUALITY E O DESEMPENHO DAS COMPANHIAS BRASILEIRAS DE CAPITAL ABERTO
}

\section{RESUMO}

O presente trabalho tem como objetivo analisar a relação entre qualidade do lucro versus desempenho das empresas brasileiras listadas na Brasil, Bolsa, Balcão (B3). A metodologia constitui-se de uma abordagem descritiva, básica e quantitativa com dados em painel estático e aplicação de regressão múltipla. Contando com uma amostra de 371 empresas brasileiras de capital aberto no período de 2010 a 2016. Foram elaboradas nove hipóteses de pesquisa pertinentes a relação entre a qualidade do lucro e o desempenho. Foram confirmadas as hipóteses de relação da persistência com o Retorno sobre o Ativo (ROA) e de relação da persistência com o Fluxo de Caixa Operacional (FCO). Conclui-se que a principal contribuição da pesquisa é a confirmação da relação positiva da proxy persistência com o desempenho das empresa no Brasil.

Palavras-chave: Qualidade dos Lucros. Desempenho. Dados em Painel.

\section{EARNING QUALITY AND PERFORMANCE OF BRAZILIAN COMPANIES OF OPEN CAPITAL}

\begin{abstract}
The present work to analyze the relationship between earning quality versus performance of Brazilian companies listed in Brazil, Bolsa, Balcão (B3). The methodology consists of a descriptive, basic and quantitative approach with static panel data and multiple regression application. With a sample of 371 Brazilian publicly traded companies from 2010 to 2016. Nine relevant research hypotheses were elaborated on the relationship between the quality of profit and performance. We confirmed the hypothesis of the relation between the persistence with Return on Asset (ROA) and the persistence relation with the Operating Cash Flow (FCO). It is concluded that the main contribution of the research is the confirmation of the positive relation of the proxy persistence with the performance of the companies in Brazil.
\end{abstract}

Keywords: Earnings Quality. Performance. Panel Date. 


\section{EARNING QUALITY Y DESEMPENÕ DE LAS EMPRESAS BRASILEÑAS DE CAPITAL ABIERTO}

\section{RESUMEN}

El presente trabajo tiene como objetivo analizar la relación entre earning quality versus desempeño de las empresas brasileñas listadas en Brasil, Bolsa, Balcón (B3). La metodología se constituye de un enfoque descriptivo, básico y cuantitativo con datos en panel estático y aplicación de regresión múltiple. Contando con una muestra de 371 empresas brasileñas de capital abierto en el período de 2010 a 2016. Se elaboraron nueve hipótesis de investigación pertinentes a la relación entre earning quality y el desempeño. Se confirmaron las hipótesis de relación de la persistencia con el Retorno sobre el Activo (ROA) y de relación de la persistencia con el Flujo de Caja Operativa (FCO). Se concluye que la principal contribución de la investigación es la confirmación de la relación positiva de la proxy persistencia con el desempeño de las empresas en Brasil.

Palabras clave: Earning Quality. Desempenõ. Datos en Panel.

\footnotetext{
1 Doutorando em Ciências Contábeis pela Universidade de Brasília - UnB. Professor de Ciências Contábeis da Universidade Federal da Grande Dourados - UFGD. Brasil. E-mail: josimarnx@yahoo.com.br

2 Doutor em Ciências Contábeis pela Universidade de Brasília - UnB. Professor da Universidade Federal da Grande Dourados - UFGD. Brasil. E-mail: rafael.mnoriller@gmail.com

3 Doutor em Controladoria e Contabilidade pela Universidade de São Paulo - USP. Professor da Universidade de Brasília - UnB. Brasil. E-mail: cesaraugustotiburciosilva@gmail.com

4 Doutor em Controladoria e Contabilidade pela Universidade de São Paulo - USP. Professor da Universidade de Brasília - UnB. Brasil. E-mail: jorgekatsumi @gmail.com
} 


\section{INTRODUÇÃO}

A qualidade da informação contábil, sobretudo do lucro, tem sido objeto de pesquisas prévias nos últimos anos (e.g., Chaney, Faccio \& Parsley, 2011; Chen, Tang, Jiang, \& Lin, 2010; Dechow, Ge, \& Schrand, 2010; Dichev, Graham, Harvey, \& Rajgopal, 2013; Iatridis, 2010; Klann \& Beuren, 2012; Oluoch, Namusonge \& Onyango, 2015; Persakis \& Iatridis, 2017; Rajgopal \& Venkatachalam, 2011; Samarasekera, 2013) em virtude da sua destacada relevância, assim como em virtude da adoção ao padrão IFRS (Iatridis, 2010; Klann \& Beuren, 2012; Silva, Bonfim, Niyama, \& Silva, 2017).

Para Dechow, Ge e Schrand (2010) a qualidade do lucro depende da relevância para a tomada de decisão do usuário da informação, em que uma maior qualidade do lucro é mais relevante para a tomada de decisão. Os lucros de alta qualidade fornecem informações mais fidedignas sobre as características de desempenho financeiro de uma firma que são relevantes para o processo de tomada de decisão pelos usuários (Dechow, Ge, \& Schrand, 2010). Em linhas gerais, lucros de alta qualidade serão melhores preditores de fluxos futuros de dividendos, e, por conseguinte, melhores preditores do valor das ações ou do valor da empresa.

As pesquisas tem relacionado qualidade do lucro com outras medidas, e.g., ao retorno anormal (Ogneva, 2012; Oluoch et al., 2015; Perotti \& Wagenhofer, 2014; Rajgopal \& Venkatachalam, 2011). Outras pesquisas têm relacionado a qualidade do lucro com o gerenciamento de resultados Chen, Tang, Jiang, \& Lin, 2010; Iatridis, 2010). E existem aquelas pesquisas que fazem associação da qualidade do lucro com o custo de capital (Aboody, Hughes, \& Liu, 2005; Francis, LaFond, Olsson, \& Schipper, 2004), com o efeito das mudanças nas normas contábeis Chen et al., 2010), com as mudanças da qualidade do lucro no tempo (Srivastava, 2014), com as práticas de governança corporativa (Fan, Wong \& Kong, 2004; Lafond \& Watts, 2008) e com o desempenho das companhias (Khavari, Didehkhani \& Garkaz, 2014).

O desempenho da empresa pode ser afetado por vários fatores (Salim \& Yadav, 2012), incluindo a qualidade da informação (e.g. Francis, Huang, Rajgopal, \& Zang, 2008). As informações sobre a empresa, sua posição financeira e seu desempenho, são úteis aos usuários na avaliação da situação atual e na confecção de estimativas futuras, em especial quanto aos fluxos de caixa futuros. No entanto, os gestores podem usar essas escolhas discricionárias de forma oportunista, seja em virtude de uma possível vinculação de sua remuneração ao resultando, assim como a outros possíveis fatores, e.g., outros contratos da companhia, ou podem usar este critério para melhorar o valor informativo da informação contábil (em especial o lucro) eventualmente para comunicar aos investidores o desempenho de longo prazo da firma (Chaney et al., 2011).

Destarte, as fraudes contábeis descobertas nos mercados de capitais ratificam a existência de falhas éticas, e a importância da transparência e da confiabilidade da informação financeira fornecida ao mercado (Lang \& Lundholm, 2000). A resposta regulatória aos escândalos financeiros tem sido tomar medidas para proteger a transparência das informações, mitigar os conflitos de interesse e garantir a independência dos auditores, tudo a fim de proteger os interesses dos investidores e aumentar a confiança dos mercados de capitais (Leuz, Nanda, \& Wysocki, 2002).

Em outras palavras, a qualidade do lucro é um fator importante para os investidores avaliarem a saúde do negócio. É um conceito multidimensional que não possui um significado único e está relacionado com a capacidade do lucro relatado em refletir o lucro real da empresa. Além disso, a qualidade do lucro mostra a estabilidade e a falta de flutuação dos lucros relatados (Khavari et al., 2014) podendo refletir no comportamento dos investidores, resultando em maiores investimentos e redução do custo de capital, melhorando o desempenho das corporações.

No entanto, diversas métricas de qualidade do lucro são apresentadas na literatura, a exemplo de Perotti e Wagenhofer (2014), de modo que, cada uma delas pode ser usada com finalidade especifica. Por sua vez, as medidas de desempenho também são diversas, entra as quais destacamos o Retorno sobre Ativo (ROA), o Retorno dobre o Patrimônio Líquido (ROE) e a geração de Fluxos de Caixa Operacional (FCO). De algum modo, pode-se relacionar as métricas de qualidade do lucro com o desempenho das companhias.

Diante do exposto e nas razões elencadas, essa pesquisa destaca a seguinte pergunta: qual a relação entre a qualidade da informação contábil, sobretudo do lucro, e o desempenho das companhias brasileiras de capital aberto? Para responder ao problema da pesquisa, esta investigação tem como objetivo analisar a relação entre qualidade do lucro versus desempenho das empresas brasileiras listadas na B3.

A motivação inicial deste estudo é a ausência de trabalhos empíricos acerca da adequação das proxies de qualidade do lucro e as medidas de desempenho, particularmente no contexto brasileiro. Poucos estudos têm focado em mercados emergentes, os quais destacam estruturas de capitais diferenciadas, sobretudo em virtude de menor pulverização dos capitais, abrindo oportunidade para o gerenciamen to de resultados, sobretudo em face dos acionistas minoritários. As pesquisas encontradas, no tocante a qualidade do lucro no contexto brasileiro, não se propuseram a relacionar métricas de qualidade do lucro e destacar a relação com medidas de desempenho das companhias. 
Esta investigação procura demonstrar qual é o comportamento daquelas, em relação aos mecanismos de desempenho das companhias brasileiras e, portanto, apontar de que forma a persistência, accruals anormais, e, coeficiente de resposta do lucro, interagem com o ROA, ROE e fluxo de caixa operacional. Por meio da utilização de regressão múltipla com dados secundários em painel contemporâneo, é possível confirmar a relação da qualidade do lucro (proxy persistência) com o desempenho (ROA e FCO) das empresas brasileiras de capital aberto. Em tempo, outras proxies Accruals Anormais e Coeficiente de Resposta dos Lucros não apresentam relação significativa a $10 \%$ com as variáveis de desempenho da pesquisa.

\section{PLATAFORMA TEÓRICA}

Em atendimento ao propósito do artigo a plataforma teórica foi dividida em três partes, (i) Qualidade do Lucro (ii) Desempenho e (iii) Desenvolvimento das Hipóteses.

\subsection{Qualidade do Lucro}

A qualidade do lucro pode ser considerada como importante mecanismo para auxiliar em uma tomada de decisão específica, considerando o desempenho da empresa (Dechow et al., 2010). Ball e Shivakumar (2005) reportam que a qualidade do lucro pode ser alterada pelo ambiente no qual a empresa está inserida e também sua classificação entre capital aberto ou fechado.

Os lucros de alta qualidade são aqueles que refletem com precisão não só o desempenho atual da empresa, mas um indicador do desempenho futuro, sendo, por consequência, uma medida do valor da empresa (Dechow \& Schrand, 2004) de modo que fornecem informações sobre as características de desempenho que são relevantes para uma decisão dos usuários (Dechow et al., 2010). Em linhas gerais, as empresas que apresentam lucros de alta qualidade forneceriam informações úteis e necessárias aos usuários da informação contábil, auxiliando-os em suas tomadas de decisões.

A qualidade do lucro responde por importante parcela das pesquisas em contabilidade, podendo ser caracterizada como a capacidade de se sustentar no futuro, i. e., o lucro atual com alta (baixa) correlação com lucros futuros teriam alta (baixa) qualidade (Lustosa, Fernandes, Nunes, \& De Araujo Junior, 2010). Já Crutchley, Jensen, e Marshall (2007) afirmam que existem diversas maneiras das empresas fraudarem seus resultados contábeis o que acaba comprometendo a qualidade do lucro das empresas, em que grandes escândalos corporativos são acompanhados por um aumento na rigidez por parte dos agentes reguladores.
Diversas pesquisas foram realizadas nos últimos anos em âmbito internacional sobre a Earnings Quality e outros fundamentos, tais como accruals (Dechow \& Dichev, 2002), informações contábeis (Caskey \& Hanlon, 2013; Dichev et al., 2013; Lawson \& Wang, 2016), auditoria (Lambert, Jones, Brazel, \& Showalter, 2017; Lennox, Wu, \& Zhang, 2016), custo de capital (Affleck-Graves, Callahan, \& Chipalkatti, 2002; Ghosh \& Moon, 2010; Karamanou \& Nishiotis, 2009; Lambert, Leuz, \& Verrecchia, 2007; Liu, Ning, \& Davidson III, 2010; Munteanu, 2011; Persakis \& Iatridis, 2017), estrutura de governança corporativa (Athanasakou \& Olsson, 2012; Choi, Lee, \& Park, 2013; Core \& Guay, 1999; El-Sayed Ebaid, 2013; Fan \& Wong, 2002; Hazarika, Karpoff, \& Nahata, 2012; Houqe \& Zijl, 2010; Jiang \& Anandarajan, 2009; Jiang, Lee, \& Anandarajan, 2008; Kent, Kent, Routledge, \& Stewart, 2016; Lin \& Hwang, 2010; Machuga \& Teitel, 2009; Sáenz González \& García-Meca, 2014; Shiri, Vaghfi, Soltani, \& Esmaeli, 2012; Shleifer \& Vishny, 1997), adoção às International Financial Reporting Standards (IFRS) (Barth, 2013; Black \& Nakao, 2017; Daske, Hail, Leuz, \& Verdi, 2008; Healy \& Palepu, 2001; Iatridis, 2010; Kajimoto \& Nakao, 2015; Klann \& Beuren, 2012; Landsman, Maydew, \& Thornock, 2012; Munteanu, 2011; Persakis \& Iatridis, 2017; Samarasekera, 2013; Silva et al., 2017; Sousa \& Galdi, 2016), disclosure (Leuz \& Verrecchia, 2000), desempenho (Khavari et al., 2014; Machdar \& Murwaningsari, 2017) e outros fatores não destacados anteriormente (Ben-Nasr, Boubakri, \& Cosset, 2015).

No que tange a relação entre qualidade do lucro e mecanismos de governança corporativa (Athanasakou \& Olsson, 2012; Choi et al., 2013; Core \& Guay, 1999; El-Sayed Ebaid, 2013; Fan \& Wong, 2002; Hazarika et al., 2012; Houqe \& Zijl, 2010; Jiang \& Anandarajan, 2009; Jian, Lee \& Anandarajan. 2008; Lin \& Hwang, 2010; Machuga \& Teitel, 2009; Sáenz González \& García-Meca, 2014; Shiri et al., 2012; Shleifer \& Vishny, 1997) as pesquisas têm evidenciado o impacto de diversos mecanismos sobre a qualidade do lucro, i.e., Institutional shareholdings, Auditor Quality, Ownership Concentration, participação nos níveis diferenciados de governança corporativa da B3 e outros.

No geral, tem sido reconhecido que esses mecanismos desempenham um papel importante em minimizar a assimetria da informação entre os insiders corporativos e investidores externos e, portanto, melhora na qualidade da informação contida nas demonstrações financeiras (Becker, Defond, Jiambalvo, \& Subramanyam, 1998; Boubraki, Bozec, Laurin, \& Rousseau, 2011; Caramanis \& Lennox, 2008; Castro \& Martinez, 2009; K. Y. . Chen, Lin, \& Zhou, 2006; Chen, 2016; Chia, Lapsley, \& Lee, 2007; De Bos \& Donker, 2004; De Miguel, Pindado, \& De La Torre, 2004; Fama \& Jensen, 1983; Gabrielsen, Gramlich, \& Plenborg, 2002; Houqe \& Zijl, 2010; Lefort, 2005; Yeo, Tan, Ho, \& Chen, 2002). A 
exemplo, Houque e Zijl (2010) destacam que os auditores externos desempenham um papel importante no acompanhamento do comportamento gerencial e garantem que a informação oferecida aos acionistas pela administração fornece uma visão justa e verdadeira da empresa.

Ainda, outras pesquisas procuram destacar a relação entre qualidade do lucro e a adoção ao padrão full IFRS (Barth, Landsman, \& Lang, 2007; Barth, Landsman, Lang, \& Williams, 2012; Bushman \& Smith, 2001; Byard, Brown, Dahya, Darrough, Davis friday \& Gore 2010; Covrig, Defond, \& Hung, 2007; Daske et al., 2008; Iatridis, 2010; Landsman et al., 2012; Li, 2010; Persakis \& Iatridis, 2017; Samarasekera, 2013; Silva et al., 2017; Tan, Wang, \& Welker, 2011). Essas pesquisas sugerem que as empresas que usam IFRS geralmente apresentam a informação contábil com maior qualidade do que àquelas que utilizam Generally Accepted Accounting Principles (GAAPs) locais (e.g., Barth et al., 2007). O aumento na qualidade melhora a comparabilidade dos valores contábeis após a adoção do padrão IFRS (Barth et al., 2012). Os benefícios ao mercado de capitais com a adoção do padrão IFRS incluem a redução do custo de capital e a melhoria da liquidez (Daske et al., 2008; $\mathrm{Li}$, 2010), o aumento do investimento estrangeiro (Covrig et al., 2007), e reduzida dispersão de previsões de analistas (Byard et al., 2010; Horton, Serafeim, \& Serafeim, 2013; Tan et al., 2011).

A redução da incerteza e da assimetria de informações facilitaria a comunicação entre os gestores e outras partes interessadas relacionadas, tais como acionistas, credores, autoridades reguladoras e de supervisão e analistas financeiros (Iatridis, 2010). Isso tenderia a reduzir os custos de agência que possam surgir (Bushman \& Smith, 2001; Healy \& Palepu, 2001), e por sua vez, conduzir a uma elevação nos retornos das ações, o que poderia estar relacionado ao desempenho financeiro atual da empresa (Gelb \& Zarowin, 2002). A adoção das IFRS fornece um sinal positivo da contabilidade em direção a uma maior qualidade e transparência (Tendeloo \& Vanstraelen, 2005).

Existem também aquelas pesquisas que apontam a relação da qualidade dos lucros com o retorno acionário, seja positivo ou negativo (Allen, Larson, \& Sloan, 2013; Bar-Yosef \& Prencipe, 2013; Chan, Chan, Jegadeesh, \& Lakonishok, 2001; Chen, Gul, \& Zhou, 2013; Francis, LaFond, Olsson, \& Schipper, 2005; Francis, Olsson, \& Schipper, 2006; Kim \& Qi, 2010; Mendonça, Costa, Galdi, \& Funchal, 2010; Mitra, 2016; Mohebbi \& Kamyabi, 2014; Namazi \& Rezaei, 2016; Perotti \& Wagenhofer, 2014; Quality, Kozlov, \& Petajisto, 2013; Yee, 2006; Zhang \& Browne, 2013).

A relação entre a qualidade do lucro e a assimetria da informação no contexto da teoria da agência também foi explorada na literatura e alguns resultados interessantes foram relatados (Namazi \&
Rezaei, 2016). Por exemplo, Bushman \& Smith (2001) descobriram que a informação de maior qualidade levaria à diminuição da assimetria de informação e poderia ser utilizada como um mecanismo de controle para monitorar as ações dos agentes com precisão, e, provavelmente reduziria os problemas de assimetria informacional entre a empresa e seus investidores, reduzindo, portanto, os custos de agência. An (2009) argumentou que a informação objetiva sobre o lucro levaria a uma maior transparência da informação financeira que, em última análise, causaria uma redução dos custos de agência. Evidências da pesquisa de Edelen, Evans, e Kadlec (2011) também sugerem o papel efetivo da transparência no fornecimento de informações do lucro sobre os custos de agência.

\subsection{Desempenho}

Diversos indicadores financeiros empresarias são utilizados para destacar o desempenho das empresas, com destaque para os indicadores de rentabilidade (Bezerra \& Corrar, 2006). Neste contexto, Ross, Westerfield e Jordan (2000) atentam para alguns indicadores de desempenho, Retorno sobre o Ativo, Retorno sobre o Patrimônio Líquido e Margem Líquida.

Em tempo, Khavari et al. (2014) expõem que o desempenho das empresas pode ser avaliado por meio de dados econômicos (por exemplo, o Valor Econômico Agregado) e também por informações contábeis e financeiras. No âmbito das informações contábeis e financeiras podem ser destacados o Dividendo por Ação, Lucro por Ação e Fluxo de Caixa Operacional (Khavari et al., 2014).

Os indicadores de desempenho oriundos das informações contábeis e financeiras são utilizados em diversas pesquisas (e.g. Barillas \& Shanken, 2018). Assim, Sahara (2013) estudou a relação do ROA com fatores macroeconômicos (Inflação, Produto Interno Bruto (PIB) e taxa de juros), encontrando relação positiva do PIB e Inflação com o ROA. Já Haniffa e Hudaib (2006) estudou a relação de fatores endógenos a empresa com o ROA, encontrando relação entre o desempenho das empresas e a governança corporativa.

Considerando fatores endógenos, poucas pesquisas têm se proposto a investigar a relação entre qualidade do lucro e desempenho (Khavari et al., 2014; Machdar \& Murwaningsari, 2017), sobretudo em economias emergentes como o Brasil. No geral, essas pesquisa apontam resultado positivo (Khavari et al., 2014; Machdar \& Murwaningsari, 2017). Em nível internacional, Khavari et al. (2014) revelam que existe uma relação significativa e positiva entre a qualidade do lucro e o desempenho das empresas. Machdar \& Murwaningsari (2017) destacam que a qualidade dos lucros afeta de forma positiva o desempenho das empresas em alguns países asiáticos. Por fim, Hoffmann e Rodrigo (2017) confirmam que em ambientes de alta assimetria informacional os 
investidores acabam não conseguindo discernir a efetiva qualidade da informação.

\subsection{Desenvolvimento das Hipóteses}

Não há uma medida geralmente aceita, mas a literatura tem desenvolvido uma variedade de proxies para a qualidade do lucro. Neste sentido, os trabalhos de Dechow e Schrand (2004), Dechow, Ge e Schrand (2010) e Perotti e Wagenhofer (2014) destacam diversas proxies, entre as quais se destacam a persistência dos lucros, accruals anormais e coeficiente de resposta do lucro, frequentemente abordadas na literatura.

Por sua vez, Dichev et al., (2013) afirmam que a qualidade dos lucros pode ser sustentada por relatórios consistentes apoiados em fluxos de caixa reais e ausência de estimativas de longo prazo, i.e., fatores não discricionários, podendo ser verificada apenas por meio de proxies, e.g., persistência e accruals anormais. Ainda, de acordo com Liu e Thomas (2000) e Dechow, Ge e Schrand (2010) outra proxy de qualidade dos lucros destacada na literatura é o Earnings Response Coefficient (ERC).

Quanto ao desempenho das empresas, pode ser apresentado por diversos indicadores, i.e., Return on Assets (ROA), Return on Equity (ROE), crescimento das vendas e também o retorno acionário. Nesse contexto, Lawson e Wang (2016) afirmam que a qualidade dos lucros possui relação como desempenho das empresas, seja no mercado financeiro, seja nos demonstrativos financeiros.

Quanto ao mercado financeiro, a pesquis a de Perotti e Wagenhofer (2014) nos EUA destaca que empresas com boa qualidade dos lucros apresentando melhor utilidade para tomada de decisão para os investidores. Já Khavani, Didehkhani e Garkaz (2014) realizaram um estudo da relação da qualidade dos lucros com o desempenho das empresas no Irã, apontando relação positiva e significativa por meio das proxies adotadas na pesquisa.

Já a pesquisa de Sloan (1996) reporta a importância da qualidade dos lucros para continuidade do desempenho das empresas, em que um alto nível de accruals geralmente resultaram em desempenhos inferiores no curto e longo prazo. $\mathrm{O}$ autor aponta, ainda, o fluxo de caixa como importante indicativo de relação com a persistência dos lucros. Por sua vez, Dechow, Ge e Schrand (2010) e Francis et al., (2008) corroboram a afirmam de que o desempenho possui relação com a qualidade dos lucros. Em linha com o contexto prévio, foram destacadas as seguintes hipóteses:

H1: Existe relação significante e positiva entre a qualidade do lucro e o ROA das companhias brasileiras de capital aberto.

H1a: Existe relação significante e positiva entre a qualidade do lucro, com base na persistência e o ROA das companhias brasileiras de capital aberto.

$\boldsymbol{H} \boldsymbol{b} \boldsymbol{b}$ : Existe relação significante e positiva entre a qualidade do lucro, com base nos accruals anormais e o ROA das companhias brasileiras de capital aberto.

H1c: Existe relação significante e positiva entre a qualidade do lucro, com base no coeficiente de resposta do lucro e o ROA das companhias brasileiras de capital aberto.

H2: Existe relação significante e positiva entre a qualidade do lucro e o ROE das companhias brasileiras de capital aberto.

H2a: Existe relação significante e positiva entre a qualidade do lucro, com base na persistência e o ROE das companhias brasileiras de capital aberto.

$\boldsymbol{H} \boldsymbol{2 b}$ : Existe relação significante e positiva entre a qualidade do lucro, com base nos accruals anormais e o ROE das companhias brasileiras de capital aberto.

$\boldsymbol{H} 2 \boldsymbol{c}$ : Existe relação significante e positiva entre a qualidade do lucro, com base no coeficiente de resposta do lucro e o ROE das companhias brasileiras de capital aberto.

H3: Existe relação significante e positiva entre a qualidade do lucro e o fluxo de caixa operacional das companhias brasileiras de capital aberto.

H3a: Existe relação significante e positiva entre a qualidade do lucro, com base na persistência e o fluxo de caixa operacional das companhias brasileiras de capital aberto. $\boldsymbol{H} 3 \boldsymbol{b}$ : Existe relação significante e positiva entre a qualidade do lucro, com base nos accruals anormais e o fluxo de caixa operacional das companhias brasileiras de capital aberto.

$\boldsymbol{H 3} \boldsymbol{c}$ : Existe relação significante e positiva entre a qualidade do lucro, com base no coeficiente de resposta do lucro e o fluxo de caixa operacional das companhias brasileiras de capital aberto.

\section{METODOLOGIA}

A pesquisa empírica, básica e predominantemente descritiva contou com uma abordagem quantitativa por meio da formulação de hipóteses estabelecidas e justificadas anteriormente, a partir de dados em painel estático, contando com informações obtidas nos demonstrativos financeiros consolidados de empresas listadas na B3, obtidas por meio da base de dados Economática ${ }^{\circledR}$. A amostra realizada de forma intencional pelo fato da B3 representar o mercado acionário brasileiro, importância das empresas de capital aberto para a economia 
nacional e pela alta liquidez dos ativos (Bernardelli \& Bernardelli, 2016).

A amostra contou com 371 empresas presentes em dez setores econômicos presentes na bolsa brasileira. Por sua vez, o período de análise contemplou os anos-calendário 2010 a 2016. Período a partir do ano de 2010 que expõe a convergência compulsória e total às International Financial Reporting Standards (full IFRS) das empresas brasileiras de capital aberto de acordo com IASB (2017). Convergência que pode comprometer a análise de períodos anteriores por enfatizar diferentes mecanismos de reconhecimento e mensuração dos

Em que:

$L L$ é igual ao lucro líquido, e foi dividido pelo ativo total no início do período $t$.

A utilização dos resíduos se justifica visto que os resultados de Dechow e Dichev (2002) demonstram uma relação negativa entre o desvio padrão dos resíduos e a persistência, e, nível dos accruals e persistência.

Para tanto, assume-se as três premissas, conforme a seguir:

1. Maior magnitude dos accruals (neste caso os resíduos da equação 1), menor qualidade do lucro (Dechow \& Dichev, 2002);

2. Menor magnitude dos accruals (neste caso os resíduos da equação 1), maior persistência (Dechow \& Dichev, 2002);

3. Relação negativa entre o desvio padrão dos resíduos e a persistência (Dechow \& Dichev, 2002). componentes dos demonstrativos financeiros, e.g., fair value.

No que tange as proxy de qualidade do lucro, a presente pesquisa toma por base os trabalhos de Dechow e Schrand (2004); Dechow et al (2010) e Perotti e Wagenhofer (2014) e foram utilizadas a persistência dos lucros, accruals anormais e coeficiente de resposta do lucros, frequentemente abordadas na literatura.

A base para mensuração do lucro é o lucro líquido $(L L)$. A métrica utilizada, - mensuração de séries temporais - a Persistência (EQ1) (ou ausência dela) é igual aos resíduos da seguinte regressão:

$$
L L_{i, t}=\alpha+\beta L L_{i, t-1}+\varepsilon_{i, t}
$$

Os resíduos são multiplicados por -1 (menos 1). Então, maior volatilidade dos resíduos da equação (1) pode ser interpretada como maior qualidade do lucro. Dessa forma, os resíduos da regressão (1) são usados nesse estudo como proxy para qualidade do lucro, em relação a persistência, e, utilizados como variável dependente na regressão OLS - equação (4) para testar a associação entre qualidade do lucro e desempenho.

No tocante a segunda métrica, accruals anormais, são estimados com base na equação 2 , e, os accruals totais são calculados como $A C C=\triangle C A$ $\triangle C L-\triangle C A S H+\triangle S T D E B T-D E P R E$, em que as variáveis são respectivamente, variação no ativo circulante, variação no passivo circulante, variação no caixa, variação na dívida de curto prazo e depreciação no exercício findo em $\mathrm{t}$.

$$
A C C_{i, t}=\alpha+\beta_{1}\left(\Delta R E V_{i, t}-\Delta A R_{i, t}\right)+\beta_{2} \Delta P P E_{i, t}+\varepsilon_{i, t}
$$

Em que:

$A C C=$ Accruals totais;

$\triangle R E V=$ Variação da receita;

$\triangle A R=$ Variação nos recebíveis ;

$\triangle P P E^{\prime}=$ Variação do Ativo Imobilizado.

Os accruals anormais, são os resíduos da equação 2, multiplicados por -1 (menos 1). Maiores valores denotam maior qualidade do lucro. A terceira métrica, earnings response coefficient (ERC) é estimado usando a seguinte regressão:

$$
R E T_{i, t}=\alpha+\beta \frac{L L_{i, t}}{P_{i, t}}+\varepsilon_{i, t}
$$

Em que:

$R E T^{\prime}=$ denota doze meses de retorno acumulado;

$P=$ Valor de mercado do patrimônio líquido. 
Dessa forma, para efeitos dessa pesquisa, o ERC são os resíduos da equação 3, multiplicado por -1 (menos 1). Dessa maneira, maiores valores dos resíduos, maior qualidade do lucro. As métricas de

Em que:

$Y_{i t}$ é a variável desempenho - ROA, ROE, Fluxo de Caixa Operacional ou Variação do Fluxo de Caixa Operacional, respectivamente para os Modelos 1, 2, 3 e 4; $\alpha$ é a constante da regressão; $\gamma$ é o coeficiente as sociado a $X_{i t}$, que são as variáveis - Persistência qualidade do lucro supracitadas são utilizadas como variáveis explanatórias da equação 4 , conforme modelo com dados em painel a seguir:

$$
Y_{i t}=\alpha+\gamma X_{i t}+u_{i t}
$$

$(P E R)$, Accruals anormais $(A C A)$ e Coeficiente de Resposta dos Lucros $(E R C) ; u_{i t}$ é o erro aleatório da regressão. Como praxe, as variáveis são resumidas e justificadas no Quadro 1.

\begin{tabular}{|c|c|c|}
\hline $\begin{array}{c}\text { Variável } \\
\text { Independente }\end{array}$ & $\begin{array}{c}\text { Sinal } \\
\text { Esperado }\end{array}$ & Justificativa \\
\hline Persistência (PER) & Positiva & $\begin{array}{l}\text { As empresas com lucros mais persistentes têm lucros/fluxo de caixa } \\
\text { mais sustentáveis que irá torná-los mais úteis na avaliação dos preços } \\
\text { das ações (Dechow et al., 2010; Perotti \& Wagenhofer, 2014). }\end{array}$ \\
\hline $\begin{array}{c}\text { Accruals Anormais } \\
\text { (ACA) }\end{array}$ & Positiva & $\begin{array}{l}\text { Os accruals anormais são indicadores de distorções induzidas, e.g., } \\
\text { sistema de mensuração imperfeito (Dechow et al., 2010; Dechow \& } \\
\text { Dichev, 2002). }\end{array}$ \\
\hline $\begin{array}{l}\text { Coeficiente de } \\
\text { Resposta dos Lucros } \\
\text { (ERC) }\end{array}$ & Positiva & $\begin{array}{l}\text { O ERC expõe que o investidor responde à informação que possui valor, } \\
\text { em que a alta correlação dos lucros com o valor implica alta qualidade } \\
\text { dos lucros (Liu \& Thomas, 1998; Dechow et al., 2010; Ghosh, Gu \& } \\
\text { Jain (2005). }\end{array}$ \\
\hline $\begin{array}{c}\text { Variável } \\
\text { Dependente }\end{array}$ & & Justificativa \\
\hline $\begin{array}{c}\text { Return on Assets } \\
\text { (ROA) }\end{array}$ & $\begin{array}{l}\text { Demerjian } \\
\text { como impo } \\
\text { lucros. }\end{array}$ & $\begin{array}{l}\text { al (2012) e Khavari, Didehkhani, e Garkaz (2014) expõem o ROA } \\
\text { te proxy de desempenho em estudos relacionados a qualidade dos }\end{array}$ \\
\hline $\begin{array}{l}\text { Return on Equity } \\
\quad(\text { ROE })\end{array}$ & $\begin{array}{l}\text { Outra } p r \\
2004)\end{array}$ & desempenho destacada na literatura e o ROE (Dechow \& Schrand, \\
\hline $\begin{array}{c}\text { Fluxo de Caixa } \\
\text { Operacional (FCO) e } \\
\text { sua variação }(\mathrm{VFCO})\end{array}$ & $\begin{array}{l}\text { Dichev et } \\
\text { dos fluxos } \\
\text { discricioná }\end{array}$ & $\begin{array}{l}\text { (2013) expõem que a qualidade dos lucros pode ser mensurada a partir } \\
\text { caixa reais e ausência de estimativas de longo prazo ou fatores } \\
\text {. }\end{array}$ \\
\hline
\end{tabular}

Quadro 1 - Justificativa das variáveis Fonte: Elaborado pelos autores.

Como limitações principais, a pesquis a foi realizada utilizando apenas o período de 2010 a 2016 e apenas empresas de capital aberto, não permitindo inferências na totalidade das empresas brasileiras. Ainda, a presente pesquisa se limita a demonstrar a qualidade do lucro com base na persistência, accruals anormais e ERC não podendo fazer maiores inferências quanto a outras proxies de qualidade dos lucros.

\section{ANÁLISE DOS RESULTADOS}

Inicialmente para analisar a relação da qualidade do lucro com o desempenho das empresas, foi utilizada como proxy para qualidade do lucro a persistência (PER), Accruals Anormais (ACA) e também o coeficiente de resposta dos lucros (ERC). Por meio da Tabela 1 é possível verificar estatísticas descritivas das variáveis adotadas na pesquisa, em que: i) o fluxo de caixa médio das empresas é de $\mathrm{R} \$$ 857.247,90; ii) os Accruals Anormais possuem um valor médio negativo de $\mathrm{R} \$ 470.470,80 \mathrm{e}$; iii) na persistência apresenta um a mediana com valor negativo de $\mathrm{R} \$ 25.523,33$ e média com valor positivo. 
Tabela 1 - Estatística descritiva (* em reais)

\begin{tabular}{|cccccc|}
\hline Variáveis & Média & Mediana & Máximo & Mínimo & Des vio Padrão \\
\hline ACA* & $-470470,80$ & 3160130 & 43805407 & $-3,92 \mathrm{e}+09$ & $1,13 \mathrm{e}+08$ \\
PER & 35390,48 & $-25523,33$ & 49041081 & -32011159 & 2630716 \\
ERC & 3,0695 & $-7,0703$ & 2745,11 & $-388,32$ & 103,27 \\
FCO* & 857247,90 & 50635,00 & $1,10 \mathrm{e}+08$ & -69668180 & 5868110 \\
VARIAÇÃO DO FCO* & 113261,38 & 636,00 & 96348510 & -144160387 & 5184800 \\
ROE & 0,0914 & 0,0871 & 0,9973 & $-0,9787$ & 0,2181 \\
ROA & 0,0152 & 0,0209 & 76,9124 & $-12,2257$ & 2,3337 \\
\hline
\end{tabular}

Obs. ROA obtido por meio da divisão do Lucro Líquido pelo Ativo Total Médio e ROE obtido por meio da divisão do Lucro Líquido pelo Patrimônio Líquido Médio.

Fonte: dados da pesquisa.

Em continuidade, a Tabela 2 destaca a matriz de correlação de Pearson e o Fator de Inflação de Variância (VIF) das variáveis independentes. Para realização do teste de multicolinearidade foi adotado o VIF e ficou constatado um valor menor do que 5, não sendo necessário ajustes nas variáveis, atendendo ao pressuposto da regressão linear. Equações 1 e 3 contando com apenas uma variável independente, portanto dispensadas do teste de multicolinearidade.

Tabela 2 - Matriz de correlação de Pearson (VIF)

\begin{tabular}{|cccc|}
\hline Equação $(2)$ & \multicolumn{2}{c}{$(\Delta \mathrm{REV}-\Delta \mathrm{AR})$} & $\Delta \mathrm{PPE}$ \\
\hline$(\Delta \mathrm{REV}-\Delta \mathrm{AR})$ & \multicolumn{2}{c}{1} & $(1,22)$ \\
$\Delta \mathrm{PPE}$ & \multicolumn{2}{c}{0,1834} & 1 \\
\hline Equação $(4)$ & $\mathrm{ACA}$ & $\mathrm{PER}$ & ERC \\
\hline ACA & 1 & $(1,03)$ & $(1,00)$ \\
PER & 0,028 & 1 & $(1,01)$ \\
ERC & $-0,0012$ & 0,006 & 1 \\
\hline
\end{tabular}

Fonte: dados da pesquisa

Por sua vez, para efeito de normalidade utilizou-se a Teoria do Limite Central, i.e., presume-se distribuição normal em amostras maiores que 100 observações (e.g. Gujarati \& Porter, 2011) diante de indícios de que os resíduos não seguem uma distribuição normal por meio do Jarque-Bera test. Ademais, foram atendidos os pressupostos de ausência de autocorrelação dos resíduos e de homocedasticidade, respectivamente, pelos testes de Durbin-Watson e Breusch-Pagan-Godfrey

Por fim, foram realizados os testes BreuschPagan e F (Chow) para detectar o melhor ajustamento do modelo não comprometendo inferências. A partir dos testes descritos anteriormente, o melhor ajustamento Randon Effects - Cross Section para o modelo 1 (variável dependente ROA), Fixed Effects Cross Section para os modelos 2 e 3 (variável dependente, respectivamente, ROE e Fluxo de Caixa Operacional) e Fixed Effects - Period (variável dependente variação do Fluxo de Caixa Operacional), para o modelo 4 .
A variável Persistência (PER) é positivamente relacionada com as variáveis de desempenho adotadas na pesquisa, não sendo significativa a $10 \%$ apenas no Modelo 2 com a variável ROE. Os Resultados confirmam as hipóteses H1a e H3a. Os achados corroboram o trabalho de Perotti e Wagenhofer (2014) e a importância da persistência no desempenho das empresas (e.g., Dechow, Ge \& Schrand, 2010).

A proxy de qualidade dos lucros Accruals anormais (ACA) apresentou relação não significativa a $5 \%$ e positiva (negativa) para o Modelo 1 (2), adotando o ROA (ROE) e não significativa a 5\% e negativa para os Modelos 3 e 4, respectivamente, FCO e variação do FCO. Assim, não suportando H1b, H2b e H3b, confirmando apenas o sinal da relação da qualidade dos lucros com o desempenho na hipótese H1b, portanto não corroborando com Richardson, Sloan, Soliman, \& Tuna (2001) e o trabalho de García-Alcober, Illueca, Prior, \& Tortosa-Ausina (2016). A justificativa para a proxy de qualidade dos lucros não apresentar relação com os indicadores de desempenho adotados na 
pesquis a pode ser o fato de considerar diferentes países na seleção da amostra das pesquisas.

Ainda, considerando a variável independente Coeficiente de Resposta dos Lucros (ERC) foi encontrada relação não significativa a $5 \%$ para os Modelos 1, 3 e 4, entretanto, positiva para o Modelos 1 e negativa para os Modelos 3 e 4. Por fim, com relação ao Modelo 2 é possível verificar relação negativa e significativa a $1 \%$, resultado com sinal da relação não esperado, justificado possivelmente pela amostra distinta das utilizadas em outras pesquisas. Destarte, inconformidade com H1c, H2c e H3c, não corroborando os achados de Ghosh, Gu e Jain (2005) e não conformidade com Park e Pincus (2001).

$$
\text { O modelo } 1 \text { apresentou } R^{2} \text { de } 0,03 \text { com a }
$$
variável ROA e no Modelo 2 com a variável dependente ROE o $R^{2}$ foi de 0,66 , portanto, maior poder explicativo. No Modelo 3 o $R^{2}$ foi de 0,95 , justificado possivelmente pela inclusão da variável dependente defasada.

Tabela 3 - Análise da regressão das variáveis de Earnings Quality (Painel Contemporâneo)

\begin{tabular}{|ccccc|}
\hline & Modelo 1 & Modelo 2 & Modelo 3 & Modelo 4 \\
\cline { 2 - 5 } Variáveis & ROA & ROE & FCO & Variação do FCO \\
\cline { 2 - 5 } & $\begin{array}{c}\text { Coeficiente } \\
(\boldsymbol{p} \text {-value })\end{array}$ & $\begin{array}{c}\text { Coeficiente } \\
(\boldsymbol{p} \text {-value })\end{array}$ & $\begin{array}{c}\text { Coeficiente } \\
(\boldsymbol{p} \text {-value })\end{array}$ & $\begin{array}{c}\text { Coeficiente } \\
(\boldsymbol{p} \text {-value })\end{array}$ \\
\hline INTERCEPTO & 0,006848 & 0,053393 & 642049 & 34945,06 \\
& $(0,9374)$ & $(0,0000)$ & $(0,0000)$ & $(0,4908)$ \\
PER & $1,90 \mathrm{e}-7$ & $2,49 \mathrm{e}-10$ & 0,2078 & 0,1914 \\
& $(0,0000)$ & $(0,9027)$ & $(0,0000)$ & $(0,0000)$ \\
ACA & $1,73 \mathrm{e}-09$ & $-0,000275$ & $-0,0027$ & $-0,00087$ \\
& $(0,7108)$ & $(0,8531)$ & $(0,5284)$ & $(0,8220)$ \\
ERC & $1.04 \mathrm{e}-05$ & $-0,000275$ & $-91,371$ & $-262,577$ \\
& $(0,9799)$ & $(0,0071)$ & $(0,9066)$ & $(0,5697)$ \\
Variável Dependente $(-1)$ & & 0,014900 & 0,4548 & $-0,1583$ \\
& & $(0,7365)$ & $(0,0000)$ & $(0,0000)$ \\
\hline$R^{2}$ & 0,0370 & 0,6685 & 0,9506 & 0,1226 \\
$R^{2}$ ajustado & 0,0341 & 0,5507 & 0,9347 & 0,1154 \\
F & 12,6492 & 48,6879 & 59,4992 & 17,1227 \\
(sig) & 0,000000 & 0,000000 & 0,000000 & 0,000000 \\
\hline
\end{tabular}

Variável Dependente: ROA (Modelo 1), ROE (modelo 2), FCO (Modelo 3) ou Variação do FCO (modelo 4). Variáveis Independentes: Persistência (PER), Accruals Anormal (ACA) e o Coeficiente de Resposta dos Lucros (ERC).

Fonte: dados da pesquisa.

\section{CONSIDERAÇÕES E RECOMENDAÇÕES}

O presente trabalho investigou a relação da qualidade dos lucros com o desempenho das empresas de capital aberto brasileiras. Persistência, Accruals Anormais e Coeficiente de Resposta dos Lucros foram as proxies de qualidade dos lucros adotadas na pesquisa. Por seu turno, ROA, ROE e Fluxo de Caixa Operacional, variáveis de desempenho adotadas na pesquisa.

Atentando para hipóteses da pesquisa foram realizadas (i) coleta de dados na Economática ${ }^{\circledR}$; (ii) seleção da amostra com a disponibilidade de dados completos para análise; (iii) aplicação do painel contemporâneo; (iv) análise e interpretação dos resultados econométricos encontrados na pesquisa.

Por meio do painel contemporâneo, os resultados apontaram relação positiva da persistência (proxy de qualidade dos lucros) como desempenho das empresas, confirmando hipóteses $H_{1 a}$ e $H_{l c}$, resultados não confirmado para proxies, ERC e Accruals Anormais. Em suma, os achados da pesquisa são correlatos com aqueles encontrados em pesquisas de nível internacional e nacional, considerando variáveis de desempenho ROA e FCO, conforme já destacado na construção das hipóteses, ceteris paribus.

Em tempo, a contribuição principal da pesquisa é a relação do desempenho com a qualidade do lucro. Apontando que a qualidade dos lucros acaba 
interferindo de forma positiva o desempenho das empresas. Ainda, cabe ressaltar que existem diversas proxies de qualidade do lucro e também diversas variáveis para mensuração do desempenho.

Diante das principais limitações da pesquisa, utilização de proxies de Qualidade do Lucro e amostra intencional, para novas pesquisas recomenda-se a utilização de outras proxies de qualidade do lucro e outras variáveis de desempenho e amostra contendo diversos países pelo mundo, e.g., G20 ou BRICS. Ainda, para novas pesquisas, seria interessante verificar possíveis divergências nos resultados por meio da segregação por setores empresariais ou por meio da segregação da amostra em países desenvolvidos e em desenvolvimento.

\section{REFERÊNCIAS}

Aboody, D., Hughes, J., \& Liu, J. (2005). Earnings Quality, Insider Trading, and Cost of Capital. Journal of Accounting Research,43(5), 651673.http://doi.org/10.1111/j.1475-679X.2005.00185.x

Affleck-Graves, J., Callahan, C. M., \& Chipalkatti, N. (2002). Earnings Predictability, Information Asymmetry, and Market Liquidity. Journal of Accounting Research,40(3), 561-583. http://doi.org/10.1111/1475-679X.00062

Allen, E. J., Larson, C. R., \& Sloan, R. G. (2013). Accrual reversals, earnings and stock returns. Journal of Accounting and Economics, 56(1), 113-129. http://doi.org/10.1016/j.jacceco.2013.05.002

An, Y. H. (2009). The Effect of Corporate Governance on Earnings Quality: Evidence from Korea's Corporate Governance Reform. RMIT University.

Athanasakou, V., \& Olsson,P. (2012). Earnings Quality, Corporate Governance, and Earnings Quality. Working Paper.

Ball, R., \& Shivakumar, L. (2005). Earnings quality in UK private firms: Comparative loss recognition timeliness. Journal of Accounting and Economics, 39(1), 83-128.

http://doi.org/10.1016/j.jacceco.2004.04.001

Bar-Yosef, S., \& Prencipe, A. (2013). The Impact of Corporate Governance and Earnings Management on Stock Market Liquidity in a Highly Concentrated Ownership Capital Market. Journal of Accounting, Auditing \& Finance, 28(3), 292-316. http://doi.org/10.1177/0148558X13492591

Barillas, F., \& Shanken, J. (2018). Comparing asset pricing models. The Journal of Finance, 73(2), 715754.
Barth, M. E. (2013). Global Comparability in Financial Reporting: What, Why, How, and When? China Journal of Accounting Studies, 1(1), 2-12. http://doi.org/10.1080/21697221.2013.781765

Barth, M. E., Landsman, W. R., \& Lang, M. H. (2007). International Accouting Standards and Accouting Quality. Journal of Accounting Research.

Barth, M. E., Landsman, W. R., Lang, M., \& Williams, C. (2012). Are IFRS-based and US GAAP-based accounting amounts comparable? Journal of Accounting and Economics, 54(1), 68-93. http://doi.org/10.1016/j.jacceco.2012.03.001

Becker, C. L., Defond, M. L., Jiambalvo, J., \& Subramanyam, K. R. (1998). The effect of audit quality on earnings management. Contemporary Accounting Research, 15(1), 1-24. http://doi.org/10.1111/j.19113846.1998.tb00547.x

Ben-Nasr, H., Boubakri, N., \& Cosset, J. C. (2015). Earnings quality in privatized firms: The role of state and foreign owners. Journal of Accounting and Public Policy, 34(4), 392-416.

http://doi.org/10.1016/j.jaccpubpol.2014.12.003

Bernardelli, L. V., \& Bernardelli, A. G. (2016). Análise sobre a Relação do Mercado Acionário com as Variáveis Macroeconômicas no Período de 2004 a 2014. Revista Evidenciação Contábil \& Finanças, 4(1), 4-17.

Bezerra, F. A., \& Corrar, L. J. (2006). Utilização da análise fatorial na identificação dos principais indicadores para avaliação do desempenho financeiro: uma aplicação nas empresas de seguros. Revista Contabilidade \& Finanças, 17(42), 50-62.

Black, R., \& Nakao, S. H. (2017). Heterogeneity in earnings quality between different classes of companies after IFRS adoption: evidence from Brazil. Revista Contabilidade \& Finanças, 28(73), 113-131. http://doi.org/10.1590/1808-057x201702750

Boubraki, N., Bozec, Y., Laurin, C., \& Rousseau, S. (2011). Incorporation Law, Ownership Structure, and Firm Value: Evidence from Canada. Journal of Empirical Legal Studies, 8(2), 358-383. Retrieved from http://www.scopus.com/inward/record.url?eid=2s2.0-

$84867676708 \&$ partnerID $=40 \&$ md 5=7ceec459271c 06d ce82109c8d1ce67b 8

Bushman, R. M., \& Smith, A. J. (2001). Financial Accounting Information and Corporate Governance. Journal of Accounting \& Economics, 23, 1-115. http://doi.org/10.1016/s0165-4101(97)00004-9 
Byard, D., Brown, A., Dahya, J., Darrough, M., Davis friday, P., \& Gore, A. (2010). The Effect of Mandatory IFRS Adoption on Financial Analysts ' Information Environment. Journal of Accounting Research, 49, 69 96.

Caramanis, C., \& Lennox, C. (2008). Audit effort and earnings management. Journal of Accounting and Economics, 45(1), 116-138.

http://doi.org/10.1016/j.jacceco.2007.05.002

Caskey, J., \& Hanlon, M. (2013). Dividend policy at firms accused of accounting fraud. Contemporary Accounting Research,30(2), 818-850. http://doi.org/10.1111/j.1911-3846.2012.01173.x

Castro, M. A. R., \& Martinez, A. L. (2009). Income smoothing, Custo de Capital de Terceiros e Estrutura de Capital no Brasil. Revista de Administração Mackenzie, 10(6), 25-46.

Chan, K., Chan, L. K., Jegadeesh, N., \& Lakonishok, J. (2001). Earnings quality and stock returns. National Bureau of Economic Research, 3, 1-23. Retrieved from http://www.nber.org/papers/w8308

Chaney, P. K., Faccio, M., \& Parsley, D. (2011). The quality of accounting information in politically connected firms. Journal of Accounting and Economics, 51(1-2), 58-76.

http://doi.org/10.1016/j.jacceco.2010.07.003

Chen, H., Tang, Q., Jiang, Y., \& Lin, Z. (2010). The Role of International Financial Reporting Standards in Accounting Quality: Evidence from the European Union. Journal of International Financial Management Accounting, 21(3), 220-278.

http://doi.org/10.1111/j.1467-646X.2010.01041.x

Chen, K. Y. ., Lin, K. ., \& Zhou, J. (2006). Audit quality and earnings management for Taiwan IPO firms. Managerial Auditing Journal, 20(1), 86-104.

Chen, L. (2016). Local Institutions, Audit Quality, and Corporate Scandals of US-Listed Foreign Firms.

Journal of Business Ethics, 351-373. http://doi.org/10.1007/s 10551-014-2370-x

Chen, S., Gul, F. A., \& Zhou, J. J. (2013). Electronic copy available at: http://ssrn.com/abstract=2719810, (October), 1-41. http://doi.org/10.1007/s 10551-0152769-z.For

Chia, Y. M., Lapsley, I., \& Lee, H.-W. (2007). Choice of auditors and earnings management during the Asian financial crisis. Managerial Auditing Journal, 22(2), 177-196. http://doi.org/10.1108/02686900710718672
Choi, B. B., Lee, D., \& Park, Y. (2013). Corporate Social Responsibility, Corporate Governance and Earnings Quality: Evidence from Korea. Corporate Governance: An International Review, 21(5), 447-467. http://doi.org/10.1111/corg.12033

Core, J., \& Guay, W. (1999). The use of equity grants to manage optimal equity incentive levels. Journal of Accounting and Economics, 28(2), 151-184. http://doi.org/10.1016/S0165-4101(99)00019-1

Covrig, V. M., Defond, M. L., \& Hung, M. (2007). Home bias, foreign mutual fund holdings, and the voluntary adoption of international accounting standards. Journal of Accounting Research , 45(1), 4170. http://doi.org/10.1111/j.1475-679X.2007.00226.x

Crutchley, C. E., Jensen, M. R. H., \& Marshall, B. B. (2007). Climate for scandal: corporate environments that contribute to accounting fraud. Financial Review, 42(1), 53-73. http://doi.org/10.1111/j.15406288.2007.00161.x

Daske, H., Hail, L., Leuz, C., \& Verdi, R. (2008). Mandatory IFRS Reporting around theWorld: Early Evidence on the Economic Consequences. Journal of Accounting Research, 46(5), 1085-1142. http://doi.org/10.1111/j.1475-679X.2008.00306.x

De Bos, A., \& Donker, H. (2004). Monitoring Accounting Changes: empirical evidences from the Netherlands. Corporate Governance, 12(1), 60-73.

De Miguel, A., Pindado, J., \& De La Torre, C. (2004). Ownership structure and firm value: New evidence from Spain. Strategic Management Journal, 25(12), 1199-1207. http://doi.org/10.1002/smj.430

Dechow, P., Ge, W., \& Schrand, C. (2010). Understanding earnings quality: A review of the proxies, their determinants and their consequences. Journal of Accounting and Economics, 50(2-3), 344401. http://doi.org/10.1016/j.jacceco.2010.09.001

Dechow, P. M., \& Dichev, I. D. (2002). The Qualityof Accruals and Earnings : The Role of Accruals Estimation Errors. The Accounting Review, 77, 35-59. http://doi.org/10.2308/accr.2002.77.s-1.61

Dechow, P. M., \& Schrand, C. M. (2004). Earnings quality. Research Foundation ofCFA Institute, 1-160. Retrieved from http://scholar.google.com/scholar?hl=en\&btnG=Search $\& q=$ intitle:Earnings +quality\#2

Demerjian, P. R., Lev, B., Lewis, M. F., \& McVay, S. E. (2012). Managerial ability and earnings quality. The Accounting Review, 88(2), 463-498. 
Dichev, I. D., Graham, J. R., Harvey, C. R., \& Rajgopal, S. (2013). Earnings quality: Evidence from the field. Journal of Accounting and Economics, 56(23), 1-33. http://doi.org/10.1016/j.jacceco.2013.05.004

Edelen, R. M., Evans, R. B., \& Kadlec, G. B. (2011). Disclosure and agency conflict: Evidence from mutual fund commission bundling. Journal of Financial Economics, 103(2), 308-326.

http://doi.org/10.1016/j.jfineco.2011.09.007

El-Sayed Ebaid, I. (2013). Corporate governance and investors' perceptions of earnings quality: Egyptian perspective. Corporate Governance: The International Journal of Business in Society, 13(3), 261-273. http://doi.org/10.1108/CG-02-2011-0011

Fama, E. F. ., \& Jensen, M. C. (1983). Separation of Ownership and Control Separation of Ownership and Control. Journal of Law and Economics, 26(2), 301325 .

Fan, J. P. H., \& Wong, T. J. (2002). Corporate ownership structure and the informativeness of accounting earnings in East Asia. Journal of Accounting and Economics, 33(3), 401-425. http://doi.org/10.1016/S0165-4101(02)00047-2

Fan, J. P. H., Wong, T. J., Administration, B., \& Kong, H. (2004). Politically-connected CEOs , Corporate Governance and Post-IPO Performance of China's Newly Partially Privatized Firms a Chinese University of Hong Kong. Corporate Governance, (December).

Francis, J., Huang, A. H., Rajgopal, S., \& Zang, A. Y. (2008). CEO reputation and earnings quality. Contemporary Accounting Research, 25(1), 109-147.

Francis, J., LaFond, R., Olsson, P. M., \& Schipper, K. (2004). Costs of equity and earnings attributes. The Accounting Review, 79(4), 967-1010. http://doi.org/10.2308/accr.2004.79.4.967

Francis, J., LaFond, R., Olsson, P., \& Schipper, K. (2005). The market pricing of accruals quality. Journal of Accounting and Economics, 39(2), 295-327. http://doi.org/10.1016/j.jacceco.2004.06.003

Francis, J., Olsson, P., \& Schipper, K. (2006). Earnings Quality. Journal of Chemical Information and Modeling,4(vol. 1), 259-340.

http://doi.org/10.1017/CBO9781107415324.004

Gabrielsen, G., Gramlich, J. D., \& Plenborg, T. (2002). Managerial ownership, information content of earnings, and discretionary accruals in a non-US setting. Journal of Business Finance and Accounting, 29(7-8), 967-988. http://doi.org/10.1111/1468-

\subsection{7}

García-Alcober, M., Illueca, M., Prior, D., \& TortosaAusina, E. (2016). Risk-taking behavior, earnings quality, and performance in Spanish banking: A profit frontier approach (No. 2016/19).

Gelb, D. S., \& Zarowin, P. (2002). Corporate disclosure policy and the informativeness of stock prices. Review of Accounting Studies, 7(1), 33-52. http://doi.org/10.1023/A:1017927530007

Ghosh, A., Gu, Z., \& Jain, P. C. (2005). Sustained earnings and revenue growth, earnings quality, and earnings response coefficients. Review of Accounting Studies, 10(1), 33-57.

Ghosh, A., \& Moon, D. (2010). Corporate debt financing and earnings quality. Journal of Business Finance and Accounting , 37(5-6), 538-559. http://doi.org/10.1111/j.1468-5957.2010.02194.x

Gujarati, D. N., \& Porter, D. C. (2011). Econometria Básica-5. AMGH Editora.

Haniffa, R., \& Hudaib, M. (2006). Corporate governance structure and performance of Malaysian listed companies. Journal of Business Finance \& Accounting, 33(7-8), 1034-1062.

Hazarika, S., Karpoff, J. M., \& Nahata, R. (2012). Internal corporate governance, CEO turnover, and earnings management. Journal of Financial Economics, 104(1), 44-69. http://doi.org/10.1016/j.jfineco.2011.10.011

Healy, P. M. ., \& Palepu, K. G. (2001). A Review of the Empirical Disclosure Literature. Journal of Chemical Information and Modeling, 53(December), 160. http://doi.org/10.1017/CBO9781107415324.004

Hoffmann, S., \& Rodrigo, P. (2017). Earnings Quality and Market Performance in LATAM Corporations: A Combined Agency and Cognitive Approach to Investors Perceptions of Managerial Information.

Horton, J., Serafeim, G., \& Serafeim, I. (2013). Does mandatory IFRS adoption improve information comparability? Contemporary Accounting Research, 30(1), 388-423. http://doi.org/10.2308/accr-50192

Houqe, N., \& Zijl, T. Van. (2010). Does Corporate Governance affect Earnings Quality: Evidence from an Emerging Market. Academy of Taiwan, ..., (2006), 112. Retrieved from http://www.researchgate.net/publication/228135032_D oes_Corporate_Governance_affect_Earnings_Quality_ Evidence_from_an_Emerging_Market/file/3deec52323 f5308a53.pdf 
Earning Quality e o Desempenho das Companhias Brasileiras de Capital Aberto

Iatridis, G. (2010). International Financial Reporting Standards and the quality of financial statement information. International Review of Financial Analysis, 19(3), 193-204.

http://doi.org/10.1016/j.irfa.2010.02.004

Jiang, W., \& Anandarajan, A. (2009). Shareholder rights, corporate governance and earnings quality. Managerial Auditing Journal, 24(8), 767-791. http://doi.org/10.1108/02686900910986402

Jiang, W., Lee, P., \& Anandarajan, A. (2008). The as sociation between corporate governance and earnings quality: Further evidence using the GOV-Score. Advances in Accounting, 24(2), 191-201. http://doi.org/10.1016/j.adiac.2008.08.011

Kajimoto, C. G. K., \& Nakao, S. H. (2015). Persistência dos Lucros Tributáveis com a Adoção das IFRS no Brasil. XII Congresso USP Iniciação Científica Em Contabilidade, 1-16.

Karamanou, I., \& Nishiotis, G. P. (2009). Disclosure and the cost of capital: Evidence from the market's reaction to firm voluntary adoption of IAS. Journal of Business Finance and Accounting , 36(7-8), 793-821. http://doi.org/10.1111/j.1468-5957.2009.02154.x

Kent, P., Kent, R. A., Routledge, J., \& Stewart, J. (2016). Choice of governance structure and earnings quality. Accounting Research Journal, 29(4), 372-390. http://doi.org/10.1108/ARJ-06-2014-0056

Khavari, M., Didehkhani, H., \& Garkaz, M. (2014). Studying the Relationship between Earning Quality and the Performance of the Firms Accepted in Tehran Stock Exchange. Advances in Environmental Biology, 8(10), 795-800.

Kim, D., \& Qi, Y. (2010). Accruals quality, stock returns, and macroeconomic conditions. Accounting Review, 85(3), 937-978.

http://doi.org/10.2308/accr.2010.85.3.937

Klann, R. C., \& Beuren, I. M. (2012). Gerenciamento de Resultados: análise comparativa de empresas brasileiras e inglesas antes e após a adoção das IFRSs. Anais Do Encontro Nacional Da Associação Nacional de Pós-Graduação E Pesquisa Em Administração, Rio de Janeiro, RJ, Brasil, 36, 1-18.

Lafond, Ryan; Watts, R. L. (2008). Earnings Smoothing, Governance and Liquidity: International Evidence. The Accounting Review, 83(2), 447-478. http://doi.org/http://dx.doi.org/10.1016/0304405X(86)90051-6

Lambert, R., Leuz, C., \& Verrecchia, R. E. (2007). Accounting Information, Disclosure, and the Cost of
Capital. Journal of Accounting Research, 45, 385-420.

Lambert, T. A., Jones, K. L., Brazel, J. F., \& Showalter, D. S. (2017). Accounting, Organizations and Society Audit time pressure and earnings quality: An examination of accelerated filings. Accounting, Organizations and Society, 58, 50-66. http://doi.org/10.1016/j.aos.2017.03.003

Landsman, W. R., Maydew, E. L., \& Thornock, J. R. (2012). The information content of annual earnings announcements and mandatory adoption of IFRS. Journal of Accounting and Economics, 53(1-2), 34-54. http://doi.org/10.1016/j.jacceco.2011.04.002

Lang, M. H., \& Lundholm, R. J. (2000). Voluntary Disclosure and Equity Offerings: Reducing Information Asymmetry or Hyping the Stock? Contemporary Accounting Research, 17(4), 623-662. http://doi.org/10.1506/9N45-F0JX-A XVW-LBW J

Lawson, B. P., \& Wang, D. (2016). The Earnings Quality Information Content of Dividend Policies and Audit Pricing. Contemporary Accounting Research, 33(4), 1685-1719. http://doi.org/10.1111/19113846.12179

Lefort, F. (2005). Ownership structure and corporate governance in Latin America.

Revista Abante, 8(1), 55-84.

Lennox, C., Wu, X., \& Zhang, T. (2016). The effect of audit adjustments on earnings quality: Evidence from China. Journal of Accounting and Economics, 61, 545562. http://doi.org/10.1016/j.jacceco.2015.08.003

Leuz, C., Nanda, D., \& Wysocki, P. D. (2002). Earnings Management and Investor Protection: An International Comparis on. Journal of Financial Economics, 69(3), 1-32. http://doi.org/10.1016/S0304405X(03)00121-1

Leuz, C., \& Verrecchia, R. E. (2000). The Economic Consequences of Increased Disclosure *. Journal of Accounting Research.

Li, S. (2010). Does Mandatory Adoption of International Financial Reporting Standards in the European Union Reduce the Cost of Equity Capital? The Accounting Review, 85, 607-636.

Lin, J. W., \& Hwang, M. I. (2010). Audit Quality, Corporate Governance, and Earnings Management: A Meta-Analysis. International Journal of Auditing, 14(1), 57-77. http://doi.org/10.1111/j.10991123.2009.00403.x

Liu, Y., Ning, Y., \& Davidson III, W. N. (2010). Earnings Management Surrounding New Debt Issues. 
Financial Review, 45(3), 659-681.

http://doi.org/10.1111/j.1540-6288.2010.00265.x

Liu, J., \& Thomas, J. (2000). Stock returns and accounting earnings. Journal of Accounting Research, 38(1), 71-101.

Lustosa, P. R. B., Fernandes, J. L. T., Nunes, D. M. S., \& De Araujo Junior, J. B. (2010). Estimativas Contábeis e Qualidade do Lucro: Análise Setorial no Brasil. Revista de Educação E Pesquisa Em Contabilidade,4(2), 43-61.

Machdar, N. M., \& Murwaningsari, E. (2017). The Effects of Earnings Quality, Conservatism, and Real Earnings Management on the Company's Performance and Information Asymmetry as a Moderating Variable. International Journal of Economics and Financial Issues, 7(2), 309-318.

Machuga, S., \& Teitel, K. (2009). Board of director characteristics and earnings quality surrounding implementation of a corporate governance code in Mexico. Journal of International Accounting, Auditing and Taxation, 18, 1-13.

http://doi.org/10.1016/j.intaccaudtax.2008.12.002

Mendonça, M. M., Costa, F. M. Da, Galdi, F. C., \& Funchal, B. (2010). O impacto da Lei Sarbanes-Oxley (SOX) na qualidade do lucro das empresas brasileiras que emitiram ADRs. Revista de Contabilidade \& Finanças, 21(52), 1-24.

Mitra, R. K. (2016). The association between earnings quality and firm-specific return volatility. Review of Accounting and Finance, 15(3), 294-316. http://doi.org/10.1108/RAF-08-2015-0100

Mohebbi, M., \& Kamyabi, Y. (2014). Product Market Structure and Earnings Quality : Evidence Tehran Stock Exchange. Asian Economic and Financial Review, 4(8), 1079-1090.

Munteanu,L. (2011). Cost of Equity, Financial Information Disclosure, and IFRS Adoption: A Literature Review. Internal Auditing \& Risk Management, 4(4), 67-80.

Namazi, M., \& Rezaei, G. (2016). The Effects of Earnings Quality Criteria on the Agency Costs: (Evidence from Tehran Stock Exchange Market). Procedia - Social and Behavioral Sciences, 230(May), 67-75. http://doi.org/10.1016/j.sbspro.2016.09.009

Ogneva, M. (2012). Accrual quality, realized returns, and expected returns: the importance of controlling for cash flow shocks. The Accounting Review, 87(4), 415444. http://doi.org/10.1073/pnas.0703993104
Oluoch, J., Namusonge, G., \& Onyango, S. (2015). Effect of accruals quality on equity security market returns: evidence from the nairobi securities exchenge. European Scientific Journal, 11(10), 132-151.

Park, C. W., \& Pincus, M. (2001). Internal versus external equity funding sources and earnings response coefficients. Review of Quantitative Finance and Accounting, 16(1), 33-52.

Perotti, P., \& Wagenhofer, A. (2014). Earnings Quality Measures and Excess Returns. Journal of Business Finance \& Accounting, 41(5-6), 545-571. http://doi.org/10.1111/jbfa.12071

Persakis, A., \& Iatridis, G. E. (2017). The joint effect of investor protection, IFRS and earnings quality on cost of capital: An international study. Journal of International Financial Markets, Institutions and Money, 46, 1-29. http://doi.org/10.1016/j.intfin.2016.10.001

Quality, E., Kozlov, M., \& Petajisto, A. (2013). Global Return Premiums on Earnings Quality, Value and Size, 1-33. http://doi.org/10.2139/s srn.2179247

Rajgopal, S., \& Venkatachalam, M. (2011). Financial reporting quality and idiosyncratic return volatility. Journal of Accounting and Economics, 51(1-2), 1-20. http://doi.org/10.1016/j.jacceco.2010.06.001

Richardson, S. A., Sloan, R. G., Soliman, M. T., \& Tuna, A. (2001). Information in Accruals about the Quality of Earnings.

Ross, S. A., Westerfield, R., Jordan, B. D., \& Firer, C. (2000). Fundamentals of corporate finance. Boston, MA: Irwin/McGraw-Hill.

Sáenz González, J., \& García-Meca, E. (2014). Does Corporate Governance Influence Earnings Management in Latin American Markets? Journal of Business Ethics, 121(3), 419-440.

http://doi.org/10.1007/s 10551-013-1700-8

Sahara, A. Y. (2013). Analis is pengaruh inflasi, suku bunga BI, dan produk domestik bruto terhadap return on asset (ROA) bank syariah di Indonesia. Sumber, 6(50), 4-60.

Salim, M., \& Yadav, R. (2012). Capital Structure and Firm Performance: Evidence from Malaysian Listed Companies. Social and Behavioral Sciences, 65(ICIBSoS), 156-166. http://doi.org/10.1016/j.sbspro.2012.11.105

Samarasekera, N. (2013). Accounting quality of UK firms under IFRS. UW A Business School. 
Shiri, M. M., Vaghfi, S. H., Soltani, J., \& Esmaeli, M. (2012). Corporate Governance and Earning Quality: Evidence from Iran. Middle-East Journal of Scientific Research, 11(6), 702-708.

Shleifer, A., \& Vishny, R. W. (1997). A survey of Corperate Governance. The Journal of Finance, Vol. LII(No. 2), 737-783.

Silva, J. P. da, Bonfim, M. P., Niyama, J. K., \& Silva, C. A. T. (2017). Adoção ao padrão IFRS e earnings quality: a persistência do lucro das empresas listadas na BM\&FBovespa. Revista de Contabilidade E Organizações, 29, 46-55.

Sousa, E. F. de; \& Galdi, F. C. (2016). The relationship between equity ownership concentration and earnings quality: evidence from Brazil. Revista de Administração, 51(4), 331-343. http://doi.org/10.1016/j.rausp.2016.07.006

Sloan, R. G. (1996). Do Stock Prices Fully Reflect Information in Accruals and Cash.

Srivastava, A. (2014). Why have measures of earnings quality changed over time? Journal of Accounting and Economics, 57(2-3), 196-217. http://doi.org/10.1016/j.jacceco.2014.04.001

Tan, H., Wang, S., \& Welker, M. (2011). Analyst following and forecast accuracy after mandated IFRS adoptions. Journal of Accounting Research, 49(5), 1307-1357. http://doi.org/10.1111/j.1475679X.2011.00422.x

Tendeloo, B. V., \& Vanstraelen, A. (2005). Earnings Management under German GAAP versus IFRS. European Accounting Review , 14(1), 155-180.

Yee, K. K. (2006). Earnings Quality and the Equity Risk Premium: A Benchmark Model. Contemporary Accounting Research,23(3), 833-877. http://doi.org/10.1506/8M44-W1DG-PLG4-8E0M

Yeo, G. H. H. ., Tan, P. M. S. ., Ho, K. W., \& Chen, S.S. (2002). Corporate Ownership Structure and the Informativeness of Earnings. Journal of Business, Finance and Accounting , 29(7), 1023-1046.

Zhang, C., \& Browne, M. J. (2013). Loss Reserve Errors, Income Smoothing and Firm Risk of Property and Casualty Insurance Companies. Annual Meeting of the American Risk and Insurance Association, Working $\mathrm{Pa}, 1-55$. 